\title{
Mukosaheilung sollte das Therapieziel sein
}

Der Integrin-Antagonist Vedolizumab induziert bei Patienten mit einer Colitis ulcerosa eine rasche Remission und zeigt auch in der Langzeittherapie eine sehr gute Wirksamkeit und Verträglichkeit. Somit bietet die Substanz eine Chance für eine klinische, endoskopische und auch histologische Remission i. S. einer „Disease clearance".

Die Colitis ulcerosa ist eine chroLebensqualität wesentlich verschlechtert. Sie führt zu einer Einschränkung der sozialen Aktivitäten und viele Patienten fühlen sich in der Ausübung ihres Berufes beeinträchtigt. „Das primäre Therapieziel ist die Besserung bzw. Remission der Symptomatik und damit die dauerhafte Normalisierung der Lebensqualität", erläuterte Dr. Peter Bossuyt, Gastroenterologe am Imelda General Hospital Bonheiden/Belgien. Primär gehe es darum, möglichst rasch eine klinische Remission zu induzieren und darüberhinaus aber auch eine endoskopische und auch histologische Remission i.S. eines "Mucosal healing" zu erreichen. Doch die Remission muss langfristig aufrechterhalten bleiben; denn nur so können strukturelle Schädigungen am Darm, Karzinome und auch operative Eingriffe vermieden werden. In klinischen Studien konnte gezeigt werden, dass eine solche „disease clearance" langfristig nisch progressive Erkrankung, die die

den Krankheitsverlauf günstig beeinflusst, d. h. weniger stationäre Behandlungen, weniger Komplikationen und weniger Operationen.

\section{Vedolizumab verspricht komplette Remission}

Die Einführung neuer zielgerichteter Therapien, welche auf einem besseren Verständnis der Immunpathogenese der Erkrankung beruht, hat die Behandlungsmöglichkeiten deutlich verbessert. „Durch den frühzeitigen Einsatz der Biologika wird das klinische Outcome und die Progression günstig beeinflusst und Kolektomien werden verhindert", so Bossuyt. Die Prävalenz der Kolektomien habe in den letzten zwanzig Jahren seit Einführung der Biologika von $10,8 \%$ auf $2,1 \%$ abgenommen. Dazu gehört Vedolizumab $\left(\right.$ Entyvio $\left.^{\circledast}\right)$. Dabei handelt es sich um einen Integrin- $\alpha_{4} \beta_{7}$-Antagonisten, d.h. die Substanz blockiert spezifisch das zelluläre Adhäsionsmolekül Integrin, was von den in die Darmwand

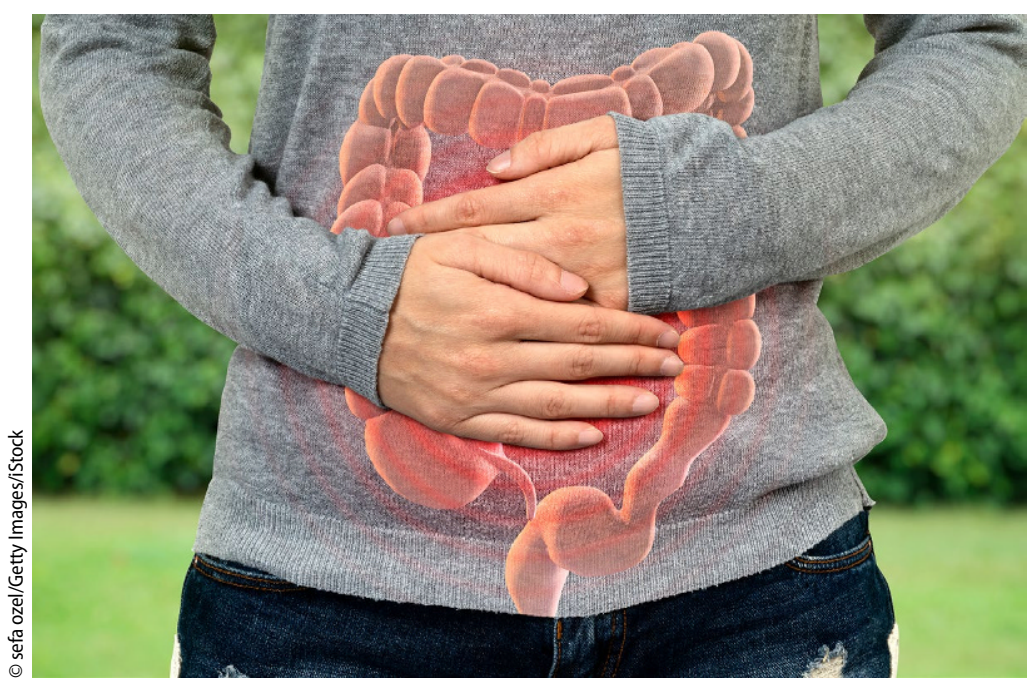

eindringenden Lymphozyten besonders stark exprimiert wird. Somit wirkt die Substanz darmselektiv. Der Benefit von Vedolizumab ist, so Bossuyt, unabhängig davon, wann die Substanz eingesetzt wird. Auch bei einer länger bestehenden Erkrankung sei nach den Ergebnissen der LOVE-UC-Studie noch eine uneingeschränkt gute Wirksamkeit garantiert [1]. Deshalb sei bei moderaten bis schweren Erkrankungen der frühe Einsatz sinnvoll.

Im Rahmen der VARSITY-Studie wurde Vedolizumab direkt mit Adalimumab verglichen. Nach 52 Wochen fand sich eine "Disease clearance" mit einer kompletten klinischen, endoskopischen und histologischen Remission bei $29,2 \%$ unter Vedolizumab vs. $16,3 \%$ unter Adalimumab [2].

\section{Autor:Dr. Peter Stiefelhagen (sti),}

Starnberg

\section{Literatur \\ 1. Vermeire S et al (2021) Gastroenterology 160(Supplement):S-91 \\ 2. Danese S et al (2021) P271. J Crohn's Colitis 15(Supplement 1):S305-S305}

Hinweis des Verlags. Der Verlag bleibt in Hinblick auf geografische Zuordnungen und Gebietsbezeichnungen in veröffentlichten Karten und Institutsadressen neutral.

J. Gastroenterol. Hepatol. Erkr. 2021 · 19:81 https://doi.org/10.1007/s41971-02100102-z

Online publiziert: 3. August 2021

(c) Springer-Verlag GmbH Austria, ein Teil von Springer Nature 2021
Quelle: Satellitensymposium „Pushing the boundaries in the management of ulcerative colitis: is "disease clearance" possible?", im Rahmen des ECCO 2021, 09.07.2021, Veranstalter: Fa. Takeda 